\title{
PERCEPÇÃO DA EQUIPE DE ENFERMAGEM DE UM SERVIÇO DE ATENDIMENTO PRÉ-HOSPITALAR MÓVEL SOBRE O GERENCIAMENTO DE ENFERMAGEM
}

\author{
Alexandre de Assis Bueno ${ }^{1}$, Andrea Bernardes ${ }^{2}$
}

\begin{abstract}
${ }^{1}$ Enfermeiro. Professor Auxiliar da Faculdade de Ciências da Saúde do Centro Universitário de Patos de Minas. Minas Gerais, Brasil. E-mail: alexisbueno@unipam.com.br

${ }^{2}$ Doutora em Enfermagem Fundamental. Professor Doutor do Departamento de Enfermagem Geral e Especializada da Escola de Enfermagem de Ribeirão Preto da Universidade de São Paulo. São Paulo, Brasil. E-mail: andreab@eerp.usp.br
\end{abstract}

RESUMO: Exigências de produtividade e qualidade ampliam os requisitos de qualificação dos trabalhadores tornando cada vez mais generalizada a implantação de modelos de formação e gestão baseados em competências profissionais, especialmente no Serviço de Atendimento Pré-Hospitalar Móvel. Esta pesquisa objetivou caracterizar o gerenciamento do enfermeiro neste serviço de acordo com a visão dos profissionais da equipe enfermagem. Trata-se de estudo exploratório, qualitativo, realizado em um município do interior de Minas Gerais. Foi utilizada entrevista semi estruturada com três enfermeiros e seis auxiliares de enfermagem e para a interpretação dos dados foi aplicada Análise Temática de Conteúdo. Os aspectos técnicos destacaram-se dentre os temas, associando o gerenciamento ao controle/ fiscalização das atividades. Evidenciou-se uma relação à distância entre equipe e supervisor, bem como a carência de educação em serviço. Espera-se que através da transformação da prática do gerenciamento de enfermagem neste serviço, ocorra aumento no conhecimento técnico-científico e melhora na qualidade da assistência.

DESCRITORES: Enfermagem. Gerenciamento. Socorro de urgência. Supervisão.

\section{EMERGENCY MEDICAL SERVICE NURSING STAFF PERCEPTIONS ABOUT NURSING MANAGEMENT}

\begin{abstract}
Demands for quality and efficiency increase worker qualification requirements, generalizing more and more the implementation of management models based on professional competence, especially in Emergency Medical Services. This study aimed to characterize nursing management in this service according to the nursing team professionals' perceptions. It is an exploratory, qualitative study carried out in a city in the interior of the Brazilian state of Minas Gerais. Semi-structured interviews among three nurses and six nursing assistants were used for data collection and Thematic Content Analysis was applied in data analysis. Technical aspects were highlighted from data analysis, associating management to control and surveillance of professional activities. A distant relationship between team and supervisor and a lack of continued education was evidenced. It is expected that a change in the nursing management practices for this service enables an increase in the technical-scientific knowledge and thus improves the quality of care.
\end{abstract}

DESCRIPTORS: Nursing. Management. Emergency relief. Supervision.

\section{LA PERCEPCIÓN DEL EQUIPO DE ENFERMERÍA DE UN SERVICIO DE ATENCIÓN HOSPITALARIA MÓVIL SOBRE LA GESTIÓN EN ENFERMERÍA}

RESUMEN: Las exigencias de productividad y calidad aumentan los requisitos de calificación de los trabajadores y la implementación de modelos de formación y de gestión basados en competencias, especialmente en el Servicio de Atención Hospitalaria Móvil. El objetivo del presente estudio fue caracterizar la administración del enfermero en este servicio según la visión de los profesionales de enfermería. Es una investigación exploratoria, cualitativa, realizada en municipio del estado de Minas Gerais. Se hicieron entrevistas semiestructuradas con tres enfermeras licenciadas y seis auxiliares de enfermería. Para el análisis de los datos se empleó el Análisis de Contenido Temático. En el análisis se destacaron los aspectos técnicos, donde se asoció la administración al control y fiscalización. Se mostró una relación a distancia entre el equipo y el supervisor, así como falta de educación en el servicio. Con la transformación de la práctica de enfermería en este servicio debe ocurrir un aumento del conocimiento técnico-científico y mejora en la calidad de la atención.

DESCRIPTORES: Enfermería. Administración. Socorro de urgencia. Supervisión. 


\section{INTRODUÇÃO}

Nas últimas décadas, o mundo tem presenciado mudanças no contexto econômico, político, social e cultural. Os serviços de saúde no Brasil apresentam uma série de deficiências cujas explicações devem ser buscadas em diferentes campos, e nesse sentido, o setor saúde vem sendo tomado como objeto de análise e de intervenção.

As exigências crescentes de produtividade e de qualidade e um contexto de mercado de trabalho instável e flexível ampliam os requisitos de qualificação dos trabalhadores e tornam cada vez mais generalizada a implantação de modelos de formação e de gestão baseados em competências profissionais. Dentre eles está o serviço de atendimento pré-hospitalar móvel de urgência e emergência, que se constitui em um importante componente de assistência à saúde.

A palavra gerência é de origem latina genere que significa o ato de gerir, administrar. A gestão, gerência ou administração, são termos que podem ser considerados sinônimos, ainda que, conjunturalmente e dependendo da situação, alguns deles se destaquem com significado mais abrangente e de maior relevância. Na Enfermagem, como nas demais áreas do saber profissional, o termo gestão tem mais aceitação, transparecendo uma incorporação da dimensão político-social e estratégica (conceitual) à operacional (técnica) da administração. ${ }^{1}$

A observação empírica mostra que o enfermeiro vem desempenhando atividades de cunho administrativo burocrático em maior escala do que aquelas relacionadas ao cuidado direto ao cliente. As tarefas são tantas que os enfermeiros normalmente não refletem acerca da sua realidade, demandando muito tempo em atividades não diretamente relacionadas aos cuidados assistenciais.

A gerência como instrumento do processo de trabalho na organização de serviços de saúde, implica na tomada de decisões que afetam a estrutura, o processo de produção e o produto de um sistema, de modo a viabilizar meios para prestação da assistência à clientela com eficiência, eficácia e efetividade. $^{2}$

Contudo, gerenciar pessoas é ainda mais complexo, pois lida com valores humanos, sentimentos, direitos/deveres. É a capacidade de liderar pessoas para fazerem o que podem e devem, otimizando o melhor do potencial humano. ${ }^{3} \mathrm{~A}$ integração entre o papel de liderança e o exercício da função de gerência pelo enfermeiro é desejada, uma vez que o líder contribui para que o envolvimento, satisfação e motivação transformem a atividade profissional dos membros da equipe de enfermagem numa atividade prazerosa, ao contrário do que vem acontecendo em muitas situações. ${ }^{4}$

As questões relativas a recursos humanos representam um componente crítico para o delineamento de novos paradigmas gerenciais para os serviços de saúde, em especial na área de enfermagem. Todos reconhecem que o desempenho de qualquer organização depende do seu pessoal.

O processo de trabalho do enfermeiro, no entanto, compõe-se de duas dimensões complementares: gerencial e assistencial. Na primeira, o enfermeiro toma como objeto à organização do trabalho e os recursos humanos em enfermagem. Na segunda, o enfermeiro toma como objeto de intervenção as necessidades de cuidado de enfermagem. Contudo, há enfermeiros com bom desempenho na assistência e frágeis na administração de enfermagem ou o inverso, o que expressa que há dificuldade de articulação entre as dimensões gerencial e assistencial. ${ }^{5}$ Tal fato contribui com a inoperância dos serviços de saúde, incluindo o Atendimento Pré-Hospitalar Móvel (APHM) que se trata de um serviço com características muito particulares.

\section{Atendimento pré-hospitalar móvel}

O termo emergência médica identifica-se com problemas de saúde que necessitam de cuidados especializados imediatos para evitar a morte ou complicações graves no indivíduo, e a urgência médica é definida como aquela situação que afeta ou coloca em perigo a saúde de uma ou de mais pessoas. ${ }^{6}$

Considerando o crescimento da demanda por serviços nesta área nos últimos anos, devido o aumento do número de acidentes e da violência urbana e a insuficiente estruturação da rede assistencial, que têm contribuído para a sobrecarga dos serviços de urgência e emergência disponibilizados para o atendimento da população, surge a necessidade de implantação do serviço de APHM.

A Portaria $\mathrm{N}^{\mathrm{o}}$ 2048/GM, de 5 de novembro de 2002, que normatiza este serviço, estabelece regras que vão desde a especialização da equipe até as características dos veículos e equipamentos a serem utilizados nas ambulâncias.

Implantado no Brasil, em setembro de 2003, o Serviço de Atendimento Móvel de Urgência (SAMU) é um serviço gratuito criado para prestar 
atendimento médico pré-hospitalar e, a depender da gravidade da situação, o paciente pode sair do domicílio, da via pública ou da unidade básica de saúde e ser encaminhado, diretamente, por meio do SAMU, para o hospital terciário. ${ }^{7-8}$

Tal serviço é caracterizado como o atendimento que procura chegar precocemente à vítima, após ter ocorrido um agravo à sua saúde (de natureza clinica, cirúrgica, traumática, inclusive psiquiátrica), que possa levar a sofrimento, sequelas ou mesmo à morte, sendo necessário prestarlhe atendimento e/ou transporte adequado a um serviço de saúde devidamente hierarquizado e integrado ao Sistema Único de Saúde. ${ }^{8}$

Este serviço móvel procura chegar à vítima nos primeiros minutos após ter ocorrido o incidente, sejam pacientes adultos, pediátricos ou gestantes, em espaços públicos ou em seus domicílios. Pode ser caracterizado como primário, quando o pedido de socorro for oriundo de um cidadão, ou secundário, quando a solicitação partir de um serviço de saúde, no qual o paciente já tenha recebido o primeiro atendimento necessário a estabilização do quadro de urgência apresentado, mas necessite ser conduzido a outro serviço de maior complexidade para a continuidade do tratamento. ${ }^{8}$

No contexto do APHM, as ações são divididas em suporte básico e suporte avançado de vida. O Suporte Básico de Vida (SBV) é definido como sendo a estrutura de apoio oferecida a pacientes com risco de mortes desconhecidas, promovidas por técnico ou auxiliar de enfermagem e um condutor/socorrista, por meio de medidas conservadoras não-invasivas, como por exemplo, a imobilização em prancha longa. Por atenderem casos de baixa complexidade, há maior número dessas viaturas, já que recebem um número mais elevado de atendimentos. Podem, ainda, atender vítimas em estado grave, como apoio às viaturas de Suporte Avançado de Vida (SAV). ${ }^{9}$

As ambulâncias de SAV funcionam como uma Unidade de Terapia Intensiva móvel, por estarem equipadas com todos os materiais necessários para atender todo tipo de vítima, classificadas em baixa, média e alta complexidade, de acordo com o agravo. A tripulação desse tipo de ambulância é composta por, pelo menos, três membros: um médico, um enfermeiro e um condutor que é capacitado para ser socorrista. ${ }^{9}$

De acordo com a portaria $\mathrm{N}^{\circ} 1863 / \mathrm{GM}$ de 29 de setembro de 2003, que institui a Política Nacional de Atenção às Urgências, para uma adequada implantação deste atendimento, o mesmo deve estar vinculado a uma Central de Regulação de Urgências e Emergências. A central deve ser de fácil acesso ao público, por via telefônica, em sistema gratuito (192 como número nacional de urgências médicas ou outro número exclusivo da saúde, se o 192 não for tecnicamente possível), onde o médico regulador, após julgar cada caso, define a resposta mais adequada, seja um conselho médico, o envio de uma equipe de atendimento ao local da ocorrência ou ainda o acionamento de múltiplos meios. O número de acesso da saúde para socorros de urgência deve ser amplamente divulgado junto à comunidade. ${ }^{8}$

\section{Gerenciamento/supervisão do atendimento pré-hospitalar móvel}

Os serviços APHM contam com equipe de profissionais de diversas áreas que devem ser habilitados pelos Núcleos de Educação em Urgências ${ }^{8}$ que têm como principal objetivo promover processo de capacitação e educação permanente dos trabalhadores para o adequado atendimento às urgências, em todos os níveis de atenção do sistema.

A qualificação das equipes e a experiência prévia na área de urgência/emergência são fatores primordiais que estão diretamente relacionados ao sucesso do atendimento, enfatizando a importância de cursos específicos para as equipes de resgate pré-hospitalar. ${ }^{10}$

A equipe de profissionais da saúde deve ser composta por: Doordenador do serviço, Responsável Técnico, Responsável de Enfermagem, Médicos Reguladores, Médicos Intervencionistas, Enfermeiros Assistenciais e Auxiliares e Técnicos de Enfermagem.

O enfermeiro deve ser profissional titular do diploma de Enfermeiro devidamente registrado no Conselho Regional de Enfermagem de sua jurisdição, habilitado para ações específicas de enfermagem, devendo além das ações assistenciais, prestar serviços administrativos e operacionais nesses sistemas de atendimento. Como requisitos gerais, ele deve ter disposição pessoal, equilíbrio emocional e autocontrole, capacidade física e mental para a atividade, disposição para cumprir ações orientadas, capacidade de trabalhar em equipe, iniciativa, facilidade de comunicação e disponibilidade para a capacitação periódica. Dentre as suas competências e atribuições estão: supervisionar e avaliar as ações da equipe de enfermagem no APHM; executar prescrições médicas por telemedicina, prestar cuidados de enfermagem 
de maior complexidade técnica a pacientes graves e com risco de morte e capacidade de tomar decisões imediatas. ${ }^{8}$

Portanto, o gerenciamento/supervisão do enfermeiro no APHM é uma atividade essencial, especialmente pelo grau de complexidade das ações, bem como pelas características peculiares desse serviço.

Frente a estes aspectos, o presente estudo justifica-se devido à natureza particular do gerenciamento realizado pelo enfermeiro que atua no APHM, uma vez que este permanece à distância. Sabe-se que a insatisfação da equipe em relação à organização do trabalho pode trazer danos potenciais para o paciente, além de dificultar as relações pessoais inter e intraprofissional. Outro aspecto de suma importância refere-se ao fato das publicações referentes a esta temática serem incipientes no Brasil, o que dificulta a organização do processo de trabalho nestes serviços.

Assim, este estudo objetiva caracterizar o gerenciamento/supervisão do enfermeiro no APHM, segundo a visão da equipe de enfermagem, em um município do interior de Minas Gerais.

\section{MÉTODO}

Trata-se de um estudo exploratório, descritivo cujo propósito foi o de observar, descrever e explorar aspectos de uma situação. ${ }^{11}$ Utilizouse uma abordagem qualitativa com o intuito de conhecer a abrangência social que nos escapa à simples observação. ${ }^{12}$

A pesquisa foi realizada no SAMU em uma cidade no interior do estado de Minas Gerais. Este município encontra-se na região do Alto Paranaíba, próxima de importantes centros produtores e consumidores do estado e de outras regiões.

A implantação do SAMU neste município se deu em setembro de 2004, com a aquisição de três ambulâncias (duas de suporte básico e uma de suporte avançado). A equipe de enfermagem é composta por 12 profissionais, sendo quatro enfermeiros e oito auxiliares de enfermagem.

Os critérios de inclusão no estudo foram: estar trabalhando na instituição no período a ser realizada a pesquisa; ser ingênuo aos propósitos do experimento, ou seja, jamais ter participado de experimentos dessa natureza e ter disponibilidade e consentir em participar.

Três enfermeiros e seis auxiliares de enfermagem com idade variando entre 25 e 53 anos de idade participaram desta pesquisa. O tempo de trabalho no APHM varia de um a 18 anos. Oito funcionários são do sexo feminino com tempo de formação variando entre um e 20 anos.

A coleta de dados foi realizada pelo pesquisador a partir da entrevista semiestruturada que combina perguntas abertas, onde o entrevistado tem a possibilidade de discorrer o tema proposto, sem resposta ou condições pré-fixadas. ${ }^{12}$ As entrevistas foram gravadas.

Os participantes foram informados sobre os objetivos da pesquisa e da garantia do anonimato, tendo liberdade total na decisão quanto à aceitação. Aqueles que optaram em participar do estudo confirmaram sua participação com a assinatura do Termo de Consentimento Livre e Esclarecido.

O trabalho foi desenvolvido de modo a garantir o cumprimento dos preceitos de Resolução 196/96 do Ministério da Saúde que normatiza a pesquisa que envolve seres humanos. Após ser autorizado pela instância superior da Prefeitura Municipal, o projeto foi encaminhado ao Comitê de Ética em Pesquisa do Centro Universitário de Araraquara, protocolo $\mathrm{N}^{\circ} 349$.

Dentre as várias técnicas propostas para análise dos dados, optou-se pela utilização da Análise Temática de Conteúdo, uma vez que este método presta-se ao estudo de motivações, atitudes, valores, crenças e tendências. ${ }^{13-14}$ As diferentes fases da análise organizam-se cronologicamente em:

1 - pré-análise: é a fase de organização que tem por objetivo de sistematizar e tornarem operacionais as idéias iniciais, de maneira a conduzir a um esquema preciso do desenvolvimento das operações sucessivas, num plano de análise.

2 - exploração do material: consiste essencialmente na operação de codificação, visando alcançar o núcleo de compreensão do texto. Referese à análise propriamente dita.

3 - tratamento dos resultados, inferência e interpretação: "[...] os resultados brutos são tratados de maneira a serem significativos ("falantes") e válidos". ${ }^{13: 101}$

A pesquisa explorou e analisou a visão dos profissionais da equipe de enfermagem com relação ao gerenciamento de enfermagem no APHM.

\section{RESULTADOS E DISCUSSÃO}

A seguir, serão reagrupadas as idéias principais de acordo com os núcleos de sentido. 


\section{Tema 1 - Controle}

Em todas as entrevistas percebemos a idéia de controle. Apesar de ser tratado de forma diferente em um e outro momento, ele sempre está presente.

[...] se o barco não tiver o marinheiro, ele afunda. É ele que comanda todas as outras pessoas. Então é igual à equipe de enfermagem... (E02).

[...] você tem que ter uma visão além do suprir, você tem que supervisionar os horários, ter uma visão ampla do que está acontecendo... (E03).

Sabe-se da presença do controle na supervisão de enfermagem e que esta "supervisão é tomada mais sobre a vertente de controle/ fiscalização" ${ }^{15: 96}$ No corpo de enfermagem, essas linhas de poder são mais marcadas quando se olha a linha vertical de comando que vai da enfermeira à auxiliar de enfermagem, mas são menos nítidas quando se olha a relação entre as enfermeiras e destas com os médicos e com a direção do hospital. ${ }^{16}$

Na realidade, essa vertente de controle está relacionada com a origem da função do supervisor que nos leva ao período do surgimento das indústrias e que se baseava no uso do poder. "Assim, o papel do elemento supervisor [...] era o de assegurar cumprimento das ordens e dos regulamentos impostos pelo seu patrão e o de garantir a produtividade". 17:117

O controle é uma etapa do processo de administração, mas manter forte controle sobre o grupo de trabalho é colocado como característica do líder autoritário. ${ }^{18}$

Evidencia-se que o controle está presente no SAMU, ainda que o enfermeiro permaneça à distância:

[...] A supervisão do SAMU, dos enfermeiros, é diferente das outras, porque aqui a gente fica separado [...] (E01).

Além da ausência física, a responsabilidade pelo gerenciamento é transferida, algumas vezes, do enfermeiro para outro membro da equipe:

[...] o médico regulador, é ele que está em contato direto com o auxiliar de enfermagem [...] (E01).

[...] porque na hora do atendimento em si, você não está com ele; o contato dele será somente com o médico regulador [...] (E02).

Apesar de tratar de um serviço com características muito peculiares, ratifica-se que o supervisor da equipe de enfermagem necessita ser enfermeiro, devendo este profissional deixar de ser o controlador à distância para ser mais um membro da equipe. Trocar "o controlar" pelo "estar na" equipe. Para que se alcance tal condição neste tipo de serviço, necessitamos superar esta estagnação do estilo gerencial utilizado em muitas instituições de saúde buscando exercer um poder baseado na cooperação entre as pessoas e no consenso, deixando de lado o "poder sobre" e valorizando o "poder de". ${ }^{19-20} \mathrm{O}$ tema controle também é evidente nas entrevistas dos auxiliares de enfermagem:

[...] o gerenciamento e supervisão dos enfermeiros, eu entendo que seja um modo assim de tá controlando a equipe de enfermagem [...] estar controlando a parte ética, técnica e a parte de trabalho em grupo [...] (AE01).

[...] porque ele é chefe, ele exige, ele pega no pé [...] (AE02).

Importante ressaltar o conceito que os auxiliares de enfermagem têm acerca do gerenciamento enquanto forma de controle e poder.

A autoridade ou o direito de comandar acompanha todas as posições de administração, sendo uma fonte de poder legítimo. Possuir poder implica a capacidade de modificar as atitudes e os comportamentos de cada indivíduo e grupo. ${ }^{18}$

Assim sendo, não podemos negar a relação direta que existe entre controle, supervisão e poder. O tipo de controle exercido pelo enfermeiro será determinado pelo tipo de poder aplicado. À medida que o poder autoritário vai sendo deixado de lado, surge a necessidade de um novo poder. Um poder que ao invés de controlar e sufocar permita o despertar, que ao invés de inibir, permita a liberdade. Um poder que ao invés de uniformizar, permita a diversidade, que ao invés de espalhar, permita a união. Desta forma, uma supervisão baseada neste poder valorizaria o ser humano em todas as suas potencialidades, além de fortalecer o senso de equipe tão necessário no APHM pelas características que ele detém.

\section{Tema 2 - Educação/capacitação da equipe}

Por diversas vezes percebemos referências dos profissionais enfermeiros em relação à capacitação da equipe.

[...] às vezes o que eu faço é depois chegar e falar: isto tá errado; não, eu não gostei. Aí eu chamo, mostro, falo que eu acho que não deve ser assim. Procuro explicar, mostrar [...] (E02).

[...] então a gente sempre faz uma consideração, um questionamento fora da hora do atendimento[...] (E01). 
[...] a nossa equipe de enfermagem passa por reciclagem. A gente tá sempre tentando melhorar o atendimento através desses treinamentos [...] (E03).

Treinar é definido como adestrar e este último como habilitar. ${ }^{21}$ Entendemos que o treinamento é algo necessário em qualquer atividade profissional, porém, percebe-se pelas falas que o tipo de treinamento predominante no serviço referido é o treinamento ocasional e não o planejado. É aquele de caráter corretivo e não profilático. Tal treinamento também é necessário, uma vez que possibilita o não incorrer em erros já cometidos, mas este se torna ineficaz no propósito de formar profissionais plenamente capacitados para o exercício de sua atividade. ${ }^{15}$

Para que haja ampliação nesta visão, há que se pensar na Educação Permanente em Serviço (EPS) que se caracteriza em uma virada no pensamento da educação profissional, no qual o processo de trabalho é valorizado como centro privilegiado da aprendizagem.

Esta parte do pressuposto da aprendizagem significativa e sugere que a transformação das práticas profissionais esteja baseada na reflexão crítica sobre as práticas reais, de profissionais reais, em ação na rede de serviços. ${ }^{22}$ A EPS tem como principais resultados a criação de espaços para a reflexão sobre o trabalho e a maior participação e responsabilização dos trabalhadores. ${ }^{23}$

Nesse ambiente de constantes imprevisibilidades em relação ao tipo de atendimento e o grau de complexidade, o conhecimento é essencial, devendo ser visto como uma dinâmica viva de produzir interpretações, significados, críticas e formas de participar da realidade. ${ }^{24}$

Todavia, os auxiliares enfatizam a importância do treinamento reiterando sua ausência no cotidiano:

[...] Eu gostaria de receber mais informação, tecnologia... eles vão em congresso fora, então, eles poderiam passar isso prá gente [...] (AE05).

[...] está bom, mas pode ser melhorado com treinamentos, isto é muito importante! Para a equipe ter um bom desempenho poderia ter mais cursos, treinamentos para o trabalho evoluir melhor [...] (AE03).

[...] todo dia você vê alguma coisa diferente e a gente nunca sabe demais. Tem sempre que tá, de nossa parte, em busca de conhecimento e tudo... a gente espera isso do enfermeiro-chefe [...] (AE02).

Ao observar tais declarações, percebe-se de maneira evidente um desejo por melhorias por parte dos auxiliares de enfermagem. Os termos utilizados por estes são limitados: treinamento, cursos, informação, mas, juntamente com estes, fica evidente um desejo maior: valorização e inserção no processo de produção como um todo.

Assim, o ser humano é visto reivindicando o papel que lhe pertence como ser criativo e inteligente, isto é, a participação não em etapas da criação, mas sim o envolvimento em todo o processo de estabelecimento de metas ao cumprimento dos propósitos. O homem em sua atividade de produção solicita permissão para não ter que deixar no relógio de ponto o seu próprio dom de criação.

Desta forma, um gerenciamento eficaz deve considerar o ser humano como um ser consciente de si mesmo e do seu papel no contexto do trabalho e da vida e uma das propostas atuais para que isso ocorra é a Educação Permanente. "[...] uma das estratégias para que isso ocorra é a educação do funcionário no seu local de trabalho. Essa estratégia facilita a transformação do potencial do empregado em comportamentos objetivos, além de oferecer condições para que interprete e utilize a realidade que o cerca $[\ldots]^{\prime 17: 147}$

Vivemos em uma época em que o estilo gerencial necessita passar por reformulação. O paradigma central da relação gerencial mudou de um poder autoritário que enfatiza o treinamento, a adaptação do outro "dentro do serviço" através da mera padronização de condutas para uma educação onde haja o "reconhecimento do outro" como ser pensante e criativo, capaz de se co-responsabilizar pela execução de suas atividades.

\section{Tema 3 - Técnicas e recursos materiais}

Este tema é abordado de forma muito semelhante pelos enfermeiros e auxiliares de enfermagem. Ficou muito evidente a importância que dão às questões relacionadas ao gerenciamento de recursos materiais.

[...] para gerenciar o serviço você tem que ter uma visão ampla do que está acontecendo... se tá faltando, se tá sobrando, onde que tem que investir mais [...] (E03).

[...] nos casos de urgência e emergência ... a gente tá sempre tentando melhorar o atendimento através dos treinamentos, suprindo com material [...] (E03).

[...] eu entendo o gerenciamento feito pelos enfermeiros [...] que um enfermeiro fica encarregado de supervisionar tudo [...] responsável por tudo o que acontece na unidade: materiais, manutenção dos equipamentos, técnicas utilizadas [...] (AE03). 
Cabe destacar a importância do controle realizado pelos profissionais no que se refere ao gerenciamento dos recursos materiais, já que o produto final do trabalho é a assistência e ela não pode sofrer interrupções, seja pela falta ou pela má qualidade de determinado material, especialmente em situações de emergência ou urgência. Assim, esse controle deve ser entendido como uma forma de possibilitar aos provedores de saúde materiais em quantidade e qualidade adequadas. Isso somente será possível através da promoção de estratégias de orientação e capacitação desses profissionais para o uso racional e otimizado desse material, bem como de uma supervisão eficiente por parte do enfermeiro.

A supervisão de enfermagem é caracterizada como uma função administrativa que envolve um processo de orientação contínua de pessoal com a finalidade de desenvolvê-lo e capacitá-lo para o serviço. ${ }^{17}$

Percebe-se, no entanto, a pouca alusão a outros aspectos importantes, como o emocional, pessoal e humano. Esta realidade é um fator preocupante, uma vez que ela nos revela uma relação fria e distante entre os membros de uma equipe. Evidencia-se claramente que a equipe de enfermagem contempla alguém que determina e alguém que executa.

Esta é a crise da enfermagem inserida na crise do trabalho em saúde que não se caracteriza como um trabalho em equipe. ${ }^{25} \mathrm{~A}$ interação entre os profissionais, inclusive com o supervisor, no atendimento aos pacientes que tem sua vida ameaçada por uma situação de urgência e emergência pode estar associada ao sentido que eles atribuem ao seu trabalho. Caso compreenda-se a ação de salvar vidas como a principal finalidade no serviço, o trabalho em equipe é altamente desejável, especialmente por que o objeto de trabalho é a pessoa vítima de agravo clínico ou traumático e o tempo para cumprir com sua finalidade é extremamente curto. ${ }^{26-28}$

Sobretudo, cabe ressaltar que tanto as atividades gerenciais em questões técnicas são essenciais para uma prestação de serviços de qualidade em qualquer ramo, especialmente na realidade do SAMU, já que há necessidade de uma resposta pronta à identificação do problema em questão.

Ao considerarem-se os princípios que norteiam a atividade gerencial, percebe-se que eles “[...] não são estáticos, neutros e absolutamente racionais, ou eminentemente técnicos... a atividade gerencial é sim extremamente dinâmica, dialética, onde as dimensões técnica, política e comunicativa estão em permanente articulação [...]". ${ }^{23: 267}$

Desta forma, faz-se necessário entender o gerente/supervisor como um agente articulador e integrador, que possa favorecer as relações interpessoais na equipe de enfermagem e também oportunizar tanto o conhecimento técnico como o científico, proporcionando um crescimento em todos os níveis.

\section{CONSIDERAÇÕES FINAIS}

A gestão é entendida como uma arte e, como tal, transcende a lógica da racionalidade, a capacidade analítica e o domínio da técnica, incorporando dimensões de criação, intuição e cidadania.

Assim, além do enfermeiro ser empreendedor, deve ser ético, voltar-se para os objetivos sociais de sua organização e/ou instituição e buscar o domínio das técnicas de gestão, agir como facilitador da busca de resultados que valorizem e dêem sentido ao seu trabalho e da equipe. Ressaltase que um gestor eficiente é aquele que busca a humanização do trabalho por intermédio de uma gestão democrática, flexível e de programas de enriquecimento pessoal dos seus atores, considerando os resultados como um trabalho coletivo.

Entende-se ser imprescindível o avanço em direção à construção plena de um modelo gerencial mais participativo, que valorize a educação permanente em saúde. A capacitação insuficiente dos recursos humanos possibilita insegurança e angústia em relação ao processo de cuidar. Os funcionários do APHM lidam com situações estressoras durante os atendimentos às vítimas $\mathrm{e}$ necessitam contar com o enfermeiro supervisor tão logo seja preciso.

Sabe-se que a supervisão deve envolver incentivo, orientação e ajuda ao pessoal, proporcionando à equipe o repensar de seus papéis e atribuiç̧̃es, tomando por referência a produção do cuidado e visando alcançar a eficiência no trabalho de enfermagem.

Para que isso seja possível, deve ser revisto o trabalho em equipe e a inserção de todos os envolvidos com vistas à consecução de um trabalho mais articulado, integrado e qualificado. As instituições devem garantir a autonomia de todos os profissionais e, simultaneamente, co-responsabilizar todos os envolvidos no processo. A administração flexível é, antes de tudo, uma filosofia de gestão e apresenta como principais características que o trabalhador deve participar ativamente do processo de trabalho, exercendo seu potencial criativo. 
Não se pode afirmar que este perfil determinaria que o serviço de APHM fosse ideal, mas, com certeza o colocaria dentro de uma perspectiva mais moderna e humanística, assim como seus gestores e trabalhadores.

Diante deste quadro, observa-se a partir deste estudo, o quanto o gerenciamento de enfermagem precisa caminhar. A caracterização do gerenciamento de enfermagem no serviço de referência para a pesquisa apresentou como resultado um perfil em que predomina o poder centralizador, com enfoque corretivo e uma atitude controladora. Ficou evidenciada uma relação distante entre a equipe e seu supervisor. Desta forma, ao supervisor fica a responsabilidade do controle e fiscalização de recursos materiais e a orientação de práticas que lhe chegam ao conhecimento de forma esporádica e não sistemática.

Ressalta-se que a mensagem enviada pelos supervisores está sendo recebida corretamente pelos subordinados. Se há alguém falando: "Eu estou te controlando", há outro que confirma: "Estou sendo controlado". Contudo, destaca-se que a parte dominada manifesta interesse por uma situação diferente, como um "companheirismo", um "envolvimento" maior, assim como um trabalho mais harmônico e conjunto.

Quase todos os profissionais percebem a necessidade de mudanças. Ainda que haja a justificativa de tratar-se de um serviço recente, em fase de adaptação, com carência de profissionais, quase todos sentem a necessidade de se alcançar melhorias em prol da qualidade da assistência.

Eis o grande desafio: transformar a prática do gerenciamento de enfermagem nos diversos campos de atuação e, em especial, no APHM. Esta atitude, certamente implicará em uma importante transformação no saber, no conhecimento, na técnica e na vivência, o que é essencial na promoção da qualidade da assistência aos clientes, como também na relação interpessoal da equipe de enfermagem. Nos modelos gerenciais mais contemporâneos o enfermeiro deve desempenhar uma gerência inovadora, buscando meios que possibilitem a melhoria da qualidade da assistência de enfermagem, maior satisfação para a equipe, bem como o alcance dos objetivos organizacionais.

\section{REFERÊNCIAS}

1. Azevedo SCO. Processo de gerenciamento $x$ gestão no trabalho do enfermeiro [dissertação]. Natal (RN): Universidade Federal do Rio Grande do Norte; 2000.
2. Passos JP, Ciosak SI. A concepção dos enfermeiros no processo gerencial em Unidade Básica de Saúde. Rev. Esc. Enferm. USP. 2006 Dez; 40(4):464-8.

3. Santos MS. Informatização de atividades administrativo-burocráticas de enfermagem relacionadas ao gerenciamento da assistência [tese]. Ribeirão Preto (SP): Universidade de São Paulo. Escola de Enfermagem de Ribeirão Preto; 2003.

4. Santos I, Castro CB. Estilos e dimensões da liderança: iniciativa e investigação no cotidiano do trabalho de enfermagem hospitalar. Texto Contexto Enferm. 2008 Dez; 17(4):734-42.

5. Hausmann M, Peduzzi M. Articulação entre as dimensões gerencial e assistencial do processo de trabalho do enfermeiro. Texto Contexto Enferm. 2009 Jun; 18(2):258-65.

6. Rodrigues FJM. Guias práticos de enfermagem em emergências. Rio de Janeiro (RJ): McGraw Hill; 2000.

7. Santos JS, Scarpelini S, Brasileiro SLL, Ferraz CA, Dallora MELV, Sá MFS. Avaliação do modelo de organização da unidade de emergência do HCFMRPUSP, adotando, como referência, as políticas nacionais de atenção às urgências e de humanização. Medicina. 2003 Abr-Dez; 36:498-515.

8. Ministério da Saúde (BR). Portaria No 2048, de 05 de novembro de 2002: Regulamento Técnico dos Sistemas de Urgência e Emergência. $3^{a}$ ed. Brasília (DF): MS; 2006.

9. Sanches S, Duarte SJH, Pontes ERJC. Caracterização das vítimas de ferimentos por arma de fogo, atendidas pelo Serviço de Atendimento Móvel de Urgência em Campo Grande-MS. Saude Soc. 2009 Mar; 18(1):95-102.

10. Fernandes RJ. Caracterização da atenção préhospitalar móvel da Secretaria da Saúde do município de Ribeirão Preto-SP [dissertação]. Ribeirão Preto (SP): Universidade de São Paulo. Escola de Enfermagem de Ribeirão Preto; 2004.

11. Polit DF, Beck CT, Hungler BP. Fundamentos de pesquisa em enfermagem. $5^{\text {a }}$ ed. Porto Alegre (RS): Artmed; 2004.

12. Minayo MCS. O desafio do conhecimento: pesquisa qualitativa em saúde. São Paulo (SP)/ Rio de Janeiro (RJ): Hucitec/Abrasco; 2000.

13. Bardin L. Análise de conteúdo. Lisboa (PT): Edições 70; 1977.

14. Minayo MCS. Interdisciplinaridade: funcionalidade ou utopia? Saúde Soc. 1994; 3(2):42-63.

15. Almeida MCP, Rocha JSY. O saber de enfermagem e sua dimensão prática. São Paulo (SP): Cortez Editora, 1986.

16. Cecílio LCO. Autonomia versus controle dos trabalhadores: a gestão do poder no hospital. Cien Saude Colet. 1999; 4(4):315-29.

17. Kurcgant $P$, Tronchin DMR, Fugulin FMT, Peres HHC, Massarollo MCKB, Fernandes MFP et al. 
Administração em Enfermagem. Rio de Janeiro (RJ): Guanabara Koogan S.A.; 2005.

18. Marquis BL, Huston CJ. Administração e liderança em Enfermagem. $4^{\text {a }}$ Ed. Porto Alegre (RS): ARTMED; 2005.

19. Bernardes A. Gestão colegiada: um desafio para a equipe multiprofissional [tese]. Ribeirão Preto (SP): Universidade de São Paulo. Escola de Enfermagem; 2005.

20. Bernardes A, Cecílio LCO, Nakao JRS, Évora YDM. Os ruídos encontrados na construção de um modelo democrático e participativo de gestão hospitalar. Cienc. Saude Colet. 2007 Jul-Ago; 12(4):861-70.

21. Bueno FS. Minidicionário da Língua Portuguesa. São Paulo (SP): Editora FTD S.A.; 1985.

22. Ministério da Saúde (BR). Política de educação e desenvolvimento para o SUS: caminhos para a educação permanente em saúde: pólos de educação permanente em saúde. Departamento de Gestão da Educação na Saúde. Brasília (DF): MS; 2004.

23. Carotta F, Kawamura D, Salazar J. Educação permanente em saúde: uma estratégia de gestão para pensar, refletir e construir práticas educativas e processos de trabalhos. Saude Soc. 2009 Jan-Mar; 18(1):48-51.

24. Pires MRGM, Spagnol CA, Brito MJM, Gazzinelli MFC, Montenegro LC. Diálogos entre a arte e a educação: uma experiência no ensino da disciplina de administração em saúde. Texto Contexto Enferm.2009 Set; 18(3):559-67.

25. Almeida MCP, Rocha SM. O trabalho de enfermagem. São Paulo (SP): Cortez Editora; 1997.

26. Garlet ER, Lima MADS, Santos JLG, Marques GQ. Organização do trabalho de uma equipe de saúde no atendimento ao usuário em situações de urgência e emergência. Texto Contexto Enferm. 2009 Jun; 18(2):266-72.

27. Pereira WAP, Lima MADS. O trabalho em equipe no atendimento pré-hospitalar à vítima de acidente de trânsito. Rev Esc Enferm USP. 2009 Jun; 43(2):320-7.

28. Galvão CM, Sawada NO, Castro AP, Corniani F. Liderança e comunicação: estratégias essenciais para o gerenciamento da assistência de enfermagem no contexto hospitalar. Rev Latino-am Enfermagem. 2000 Out; 8(5):34-43.
Correspondência: Andrea Bernardes

Escola de Enfermagem de Ribeirão Preto da Universidade de São Paulo

Avenida Bandeirantes, 3900

14040-902 - São Paulo, SP, Brasil

E-mail: andreab@eerp.usp.br
Recebido em: 9 de setembro de 2008 Aprovação final: 7 de novembro de 2009 